\title{
Globally optimized Fourier finite-difference method for ultrasound breast imaging
}

\author{
Lianjie Huang ${ }^{a}$, Kenneth M. Hanson ${ }^{a}$, Youli Quan ${ }^{b}$, Cuiping $\mathrm{Li}^{c}$, and Neb Duric ${ }^{c}$ \\ ${ }^{a}$ Los Alamos National Laboratory, Los Alamos, NM 87545 \\ ${ }^{b}$ Stanford University, Stanford, CA 94305 \\ ${ }^{b}$ Karmanos Cancer Institute, Detroit, MI 48201
}

\begin{abstract}
Ultrasound reflection imaging is a promising imaging modality for detecting small, early-stage breast cancers. Properly accounting for ultrasound scattering from heterogeneities within the breast is essential for high-resolution and high-quality ultrasound breast imaging. We develop a globally optimized Fourier finite-difference method for ultrasound reflectivity image reconstruction. It utilizes an optimized solution of acoustic-wave equation and a heterogeneous sound-speed distribution of the breast obtained from tomography to reconstruct ultrasound reflectivity images. The method contains a finite-difference term in addition to the split-step Fourier implementation, and minimizes ultrasound phase errors during wavefield inward continuation while maintaining the advantage of high computational efficiency. The accuracy analysis indicates that the optimized method is much more accurate than the split-step Fourier method. The computational efficiency of the optimized method is one to two orders of magnitude faster than time-reversal imaging using a finite-difference time-domain wave-equation scheme. Our new optimized method can accurately handle ultrasound scattering from breast heterogeneities during reflectivity image reconstruction. Our numerical imaging examples demonstrate that the optimized method has the potential to produce high-quality and high-resolution ultrasound reflectivity images in combination with a reliable ultrasound sound-speed tomography method.
\end{abstract}

Keywords: Breast heterogeneities, finite difference, Fourier, image reconstruction, optimized, reflectivity, sound speed, ultrasound breast imaging, ultrasound scattering.

\section{INTRODUCTION}

High-quality and high-resolution images are critically important for early breast cancer detection and diagnosis. Clinical studies have shown that ultrasound imaging has tremendous potential to detect small, early-stage breast cancers that cannot be detected by X-ray mammography. ${ }^{1-3}$ In addition, ultrasound breast imaging is one of the most promising screening tools as an alternative to X-ray mammography. ${ }^{2}$ The primary limitation of ultrasound imaging is due to its low resolution and noisy images (full of speckles), because it does not properly account for ultrasound scattering from breast heterogeneities during reflectivity image reconstruction. Sound speeds and densities of breast tissue are inhomogeneous, and those of tumors are different from the surrounding tissues. These differences in mechanical properties result in ultrasound scattering, particularly in dense breasts. With the development of new circular ultrasound arrays for clinical breast imaging, ${ }^{4-10}$ heterogeneous sound-speed distributions of the breast can be reliably reconstructed using ultrasound tomography. ${ }^{11-16}$ Ultrasound reflection images can be significantly improved by using the heterogeneous sound-speed distribution of the breast during reflectivity image reconstruction. The split-step Fourier method was recently introduced to ultrasound imaging and it can approximately account for ultrasound scattering. ${ }^{17}$ It is computationally about two order of magnitude faster than time-reversal ultrasound imaging using a finite-difference time-domain wave-equation scheme. ${ }^{18}$

We develop a globally optimized Fourier-difference method to accurately handle ultrasound scattering while maintaining high computational efficiency during ultrasound reflectivity image reconstruction. The method is based on the formal solution of a one-way wave equation in heterogeneous media governing ultrasound propagation in the breast. It is optimized for the entire range of sound-speed perturbations within the breast to minimize ultrasound phase errors during wavefield inward continuation. The accuracy analysis shows that the optimized method significantly improves the imaging accuracy compared to the split-step Fourier method. The optimized method performs one additional step of ultrasound

Send correspondence to Lianjie Huang: 1jh@lanl.gov

Medical Imaging 2008: Ultrasonic Imaging and Signal Processing, edited by Stephen A. McAleavey, Jan D'hooge, Proc. of SPIE Vol. 6920, 692007, (2008) · 1605-7422/08/\$18 · doi: 10.1117/12.771024

Proc. of SPIE Vol. $6920692007-1$ 
scattering compensation during each recursive step of inward continuation of ultrasound wavefields, in addition to the splitstep Fourier implementation. It is still computationally much more efficient than the finite-difference-based time-reversal imaging method. ${ }^{18}$ Like the split-step Fourier method, the optimized method is implemented in the frequency-space and frequency-wavenumber domains using Fourier transforms to minimize the numerical dispersion. In homogeneous regions, it leads to the phase-shift imaging method. ${ }^{19}$ We use synthetic ultrasound breast data to demonstrate that the globally optimized Fourier finite-difference method can accurately account for ultrasound scattering from breast heterogeneities, and has great potential to produce high-resolution and high-quality clinical breast images.

\section{METHODOLOGY}

Ultrasound scattering from breast heterogeneities cannot be accurately described using ray theory, on which most of current ultrasound imaging algorithms are based. Ultrasound wave propagation in the breast is governed by the acoustic-wave equation in heterogeneous media. To properly and accurately account for ultrasound scattering during breast imaging, it is necessary to develop an imaging algorithm based on wave theory.

The acoustic-wave equation can be decomposed into two one-way wave equations describing wave propagation in opposite directions. One of these one-way wave equations in the frequency-space domain is

$$
\frac{\partial U(x, z ; \omega)}{\partial z}=-i Q(x, z ; \omega) U(x, z ; \omega),
$$

where $U$ is the ultrasound wavefield, and $Q$ is an operator defined by

$$
Q \equiv \sqrt{\frac{\omega^{2}}{c^{2}(x, z)}+\frac{\partial^{2}}{\partial x^{2}}}=\frac{\omega}{c(x, z)} R,
$$

where $\omega$ is the circular frequency, $(x, z)$ is the space location, $c$ is the sound speed, and $R$ is the square-root operator given by

$$
R \equiv \sqrt{1-X^{2}}
$$

with

$$
X^{2} \equiv-\frac{c^{2}}{\omega^{2}} \frac{\partial^{2}}{\partial x^{2}}
$$

The formal solution of Eq. (1) is

$$
U(x, z+\Delta z ; \omega)=\exp \left\{-i \int Q d z\right\} U(x, z ; \omega),
$$

which extrapolates ultrasound wavefield $U$ from the depth level at $z$ to the next depth level at $z+\Delta z$.

To numerically solve Eq. (5), we rewrite Eq. (2) as

$$
Q=\frac{\omega}{c_{0}} \sqrt{1-X_{0}^{2}}+\left\{\frac{\omega}{c} \sqrt{1-X^{2}}-\frac{\omega}{c_{0}} \sqrt{1-X_{0}^{2}}\right\}
$$

where $c_{0}$ is the sound speed of a reference medium, and $X_{0}^{2}$ is given by

$$
X_{0}^{2}=-\frac{c_{0}^{2}}{\omega^{2}} \frac{\partial^{2}}{\partial x^{2}}=\frac{X^{2}}{m^{2}}
$$

where $m$ is the sound-speed contrast

$$
m(x, z) \equiv \frac{c(x, z)}{c_{0}(z)}
$$

which is the reciprocal of the refraction index. We expand the square-root operator $R$ in the form

$$
R \approx 1-\frac{a X^{2}}{1-b X^{2}}
$$




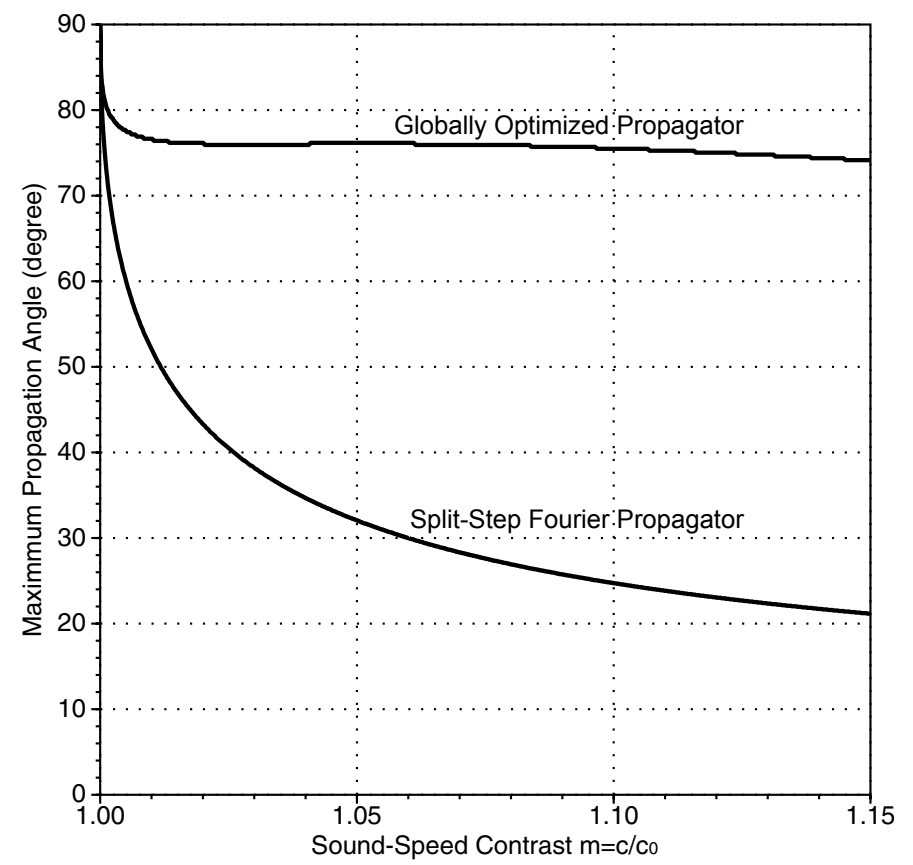

Figure 1: The maximum propagation angle within $1 \%$ of phase error versus the sound-speed contrast for the globally optimized Fourier finite-difference method and the split-step Fourier method. The maximum propagation angle for the optimized method is much larger than the split-step Fourier method.

where $a$ and $b$ are free coefficients. Making use of Eq. (9), Eq. (6) becomes

$$
Q \approx \sqrt{\frac{\omega^{2}}{c_{0}^{2}}+\frac{\partial^{2}}{\partial x^{2}}}+\frac{\omega}{c_{0}}\left(\frac{1}{m}-1\right)-\frac{\omega}{c_{0}} \frac{a(m-1) X_{0}^{2}}{1-b\left(1+m^{2}\right) X_{0}^{2}} .
$$

The ultrasound reflection imaging scheme using the formal solution (5) with the the first two terms of Eq. (10) for inward wavefield continuation is the split-step Fourier method. ${ }^{17}$

The phase error of ultrasound wavefield is zero along the primary inward continuation direction, then increases with increasing the propagation angle relative to the primary direction. We substitute Eq. (10) into Eq. (5), and minimize the phase error for the entire range of sound-speed perturbations within the breast to obtain the optimized coefficients $a$ and $b$. The formal solution (5) with the third term of Eq. (10) is implemented using an implicit finite-difference scheme. The ultrasound reflection imaging approach using the formal solution (5) and Eq. (10) with optimized coefficients $a$ and $b$ for inward wavefield continuation is termed the globally optimized Fourier finite-difference method.

\section{PHASE ACCURACY}

We conduct accuracy analysis of the globally optimized Fourier finite-difference method, and compare it with that of the split-step Fourier method. Assuming that the maximum sound-speed perturbation within the breast is 15\%, Fig. 1 depicts the relationships of the maximum propagation angle within $1 \%$ of phase error versus the sound-speed contrast for the globally optimized Fourier finite-difference method and the split-step Fourier method. It shows that the optimized method can accurately handle much larger propagation angles than the split-step Fourier method. In other words, the optimized method is much more accurate than the split-step Fourier method for a given propagation angle. It is important to develop an imaging method capable of handling large-angle propagation for high-resolution ultrasound imaging because cancers have higher sound-speeds and densities compared to their surrounding tissues, and they generate scattered ultrasound waves that propagate along a wide range of angle. The accuracy analysis in Fig. 1 shows that the split-step Fourier method has less than $1 \%$ of phase error within a propagation angle of approximately $40^{\circ}-50^{\circ}$ centered around the primary backpropagation direction. The propagation angle increases to approximately $150^{\circ}$ for the globally optimized Fourier finite-difference method. For regions with a large sound-speed contrast, this angle is nearly 3.5 times larger than that for the split-step Fourier method. 


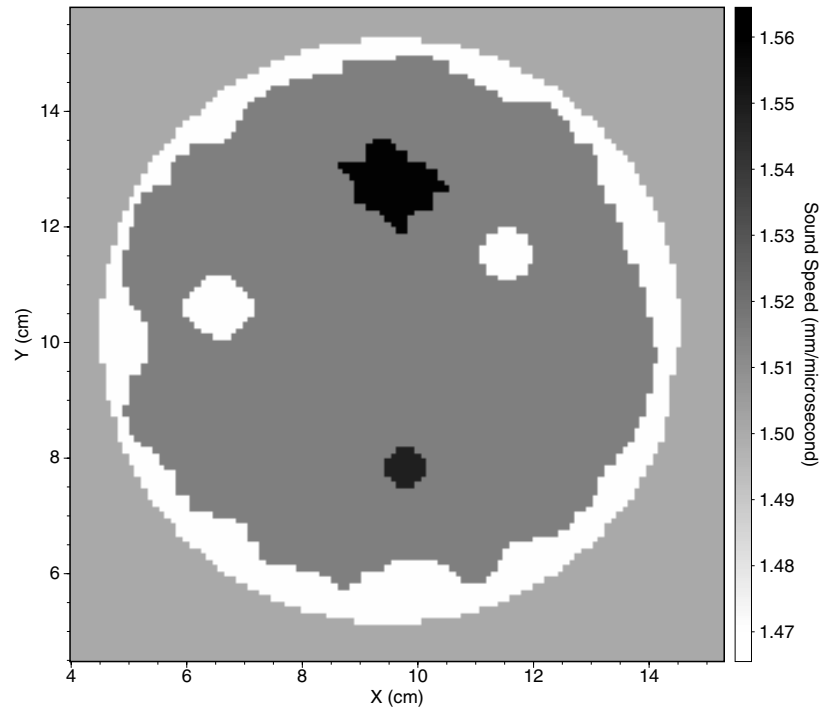

(a) Sound speed of a numerical breast phantom with four anomalies

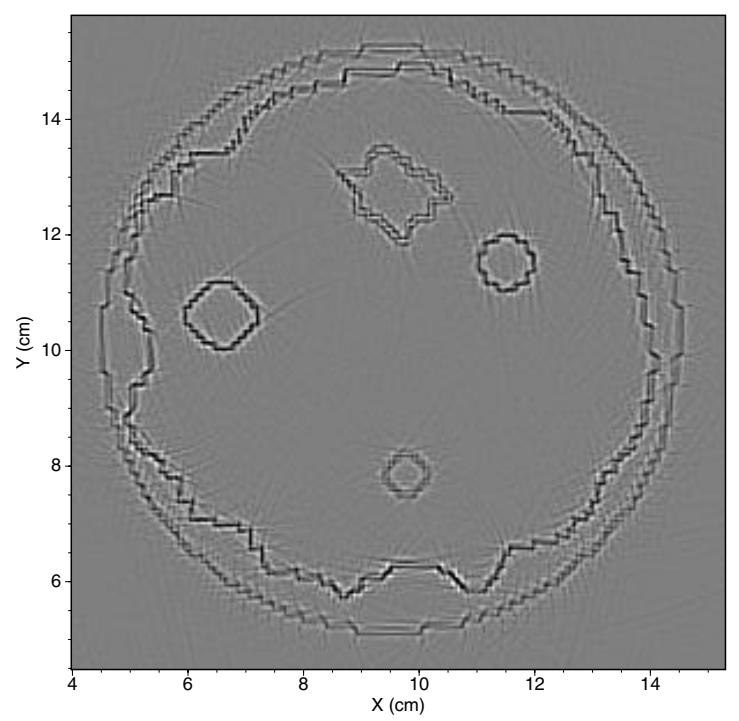

(c) Image obtained with the phase-shift method and a uniform sound-speed

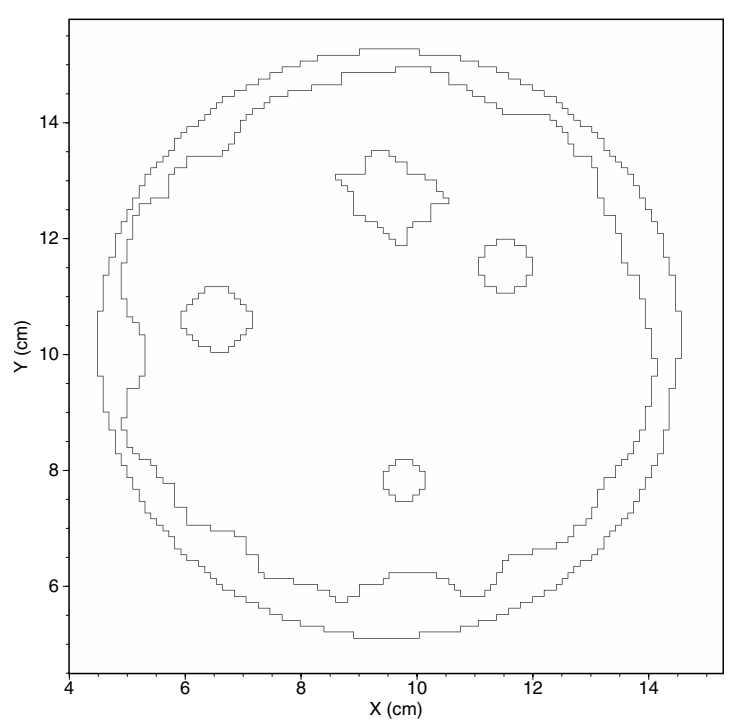

(b) Reflectivity of the phantom in (a)

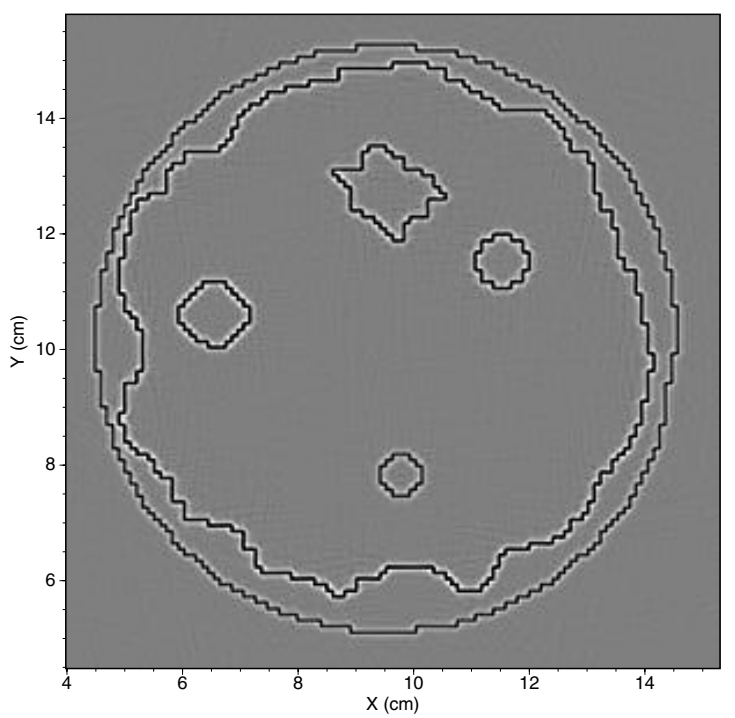

(d) Image obtained with the optimized method and the heterogeneous sound-speed in (a)

Figure 2: (a) Sound speed of a numerical breast phantom with four anomalies. (b) Reflectivity within the phantom in (a) shows where changes of acoustic impedance occur. (c) Reflectivity image reconstructed using the phase-shift method and a uniform sound-speed. (d) Image produced using the globally optimized Fourier finite-difference method and the heterogeneous sound-speed of the phantom in (a).

\section{NUMERICAL IMAGING EXAMPLES}

We use two numerical breast phantoms to validate the capability of the globally optimized Fourier finite-difference method for high-resolution ultrasound reflection imaging. One is constructed from an experimental breast phantom containing four mimic breast anomalies, and another is derived from an ultrasound sound-speed tomography image of an in-vivo breast with a cancer. A finite-difference time-domain wave-equation scheme is used to compute synthetic ultrasound pulse-echo reflection data for a ring transducer array surrounding the phantoms. The transducers are equally distributed along the ring with a diameter of $20 \mathrm{~cm}$. The central frequency of the data is $1 \mathrm{MHz}$. The reflection images reconstructed using the exact sound-speed distributions have the best image quality and highest image resolution that could be achieved. They are used to compare with reflection images produced using tomography sound-speed models to study the effects of inaccurate 


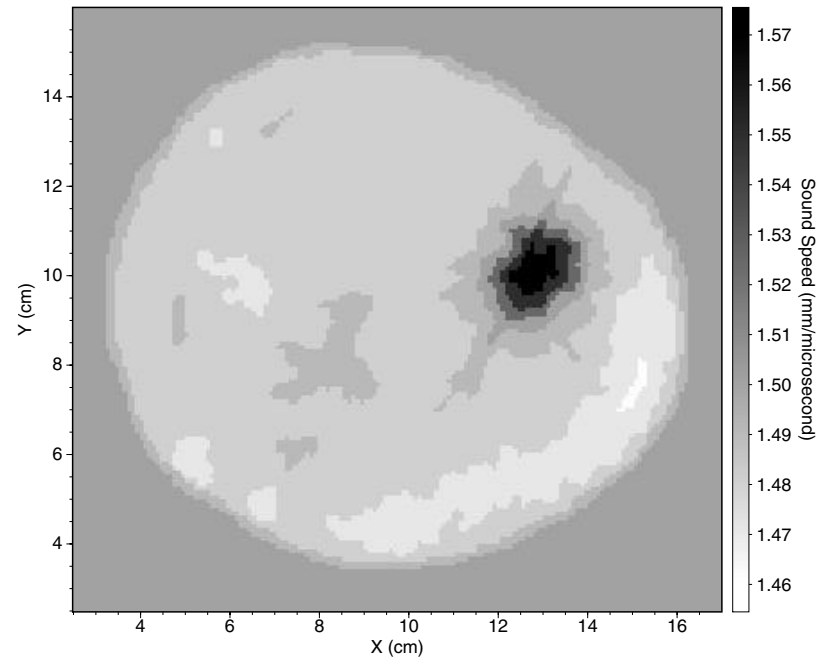

(a) Sound speed of a numerical breast phantom derived from invivo ultrasound data

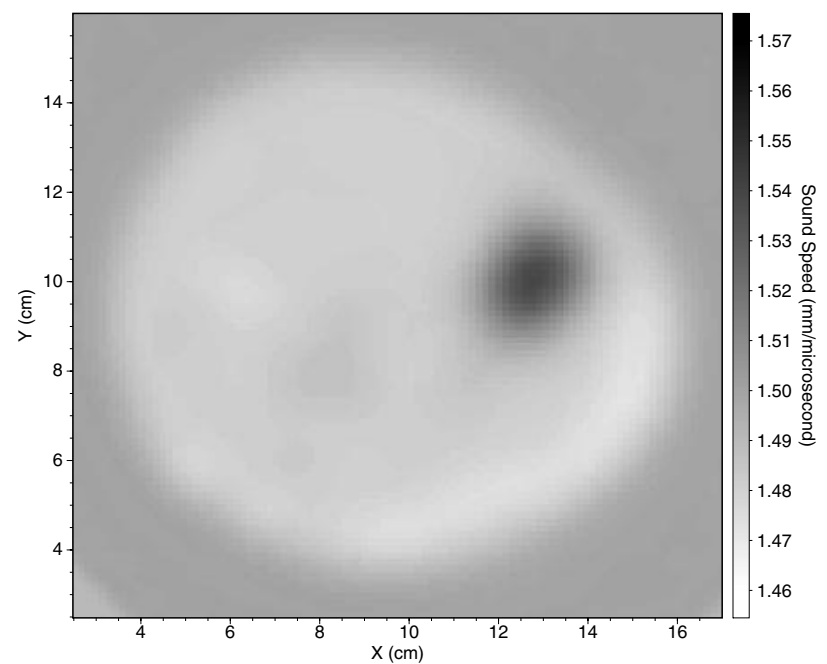

(c) Sound-speed tomography with two iterations

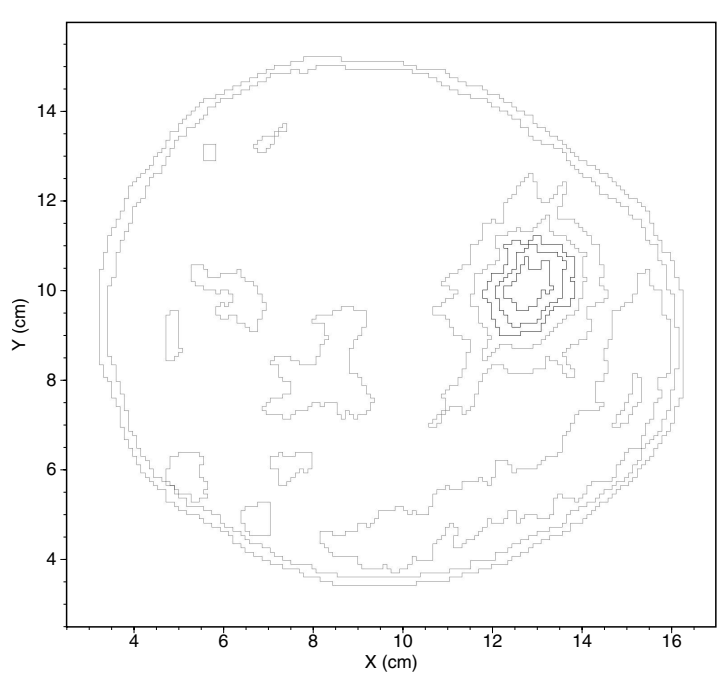

(b) Reflectivity of the phantom in (a)

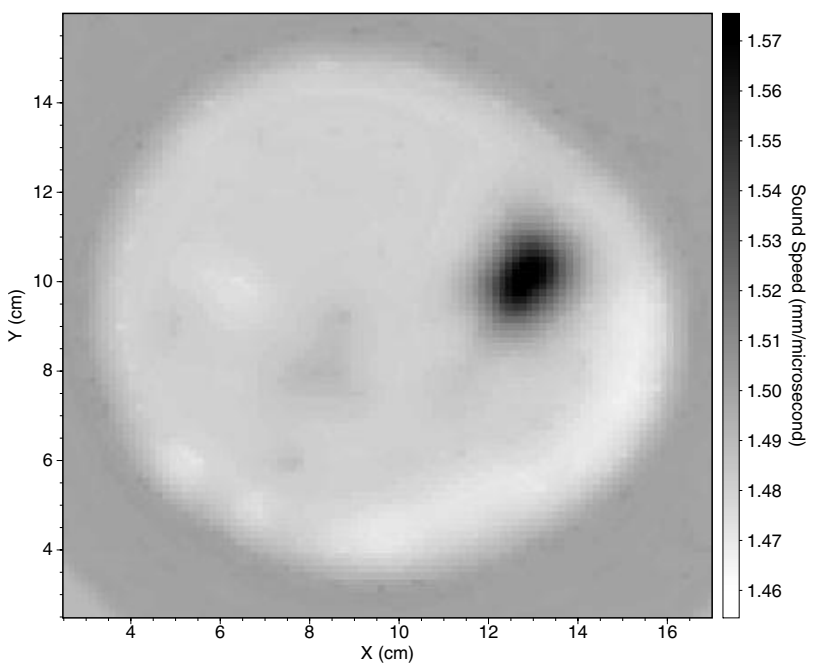

(d) Sound-speed tomography with ten iterations

Figure 3: (a) Sound speed of a numerical breast phantom derived from a sound-speed tomography result of an in-vivo ultrasound breast dataset. The region with higher sound speeds is a cancer. (b) Reflectivity within the phantom in (a) shows where changes of acoustic impedance occur. Sound-speed transmission tomography results of the phantom reconstructed with two iterations (c) and ten iterations (d).

sound-speed distributions on reflectivity image reconstruction.

Figure 2(a) is the sound-speed distribution of the numerical breast phantom containing two mimic tumors with high sound-speeds, and two mimic fatty tissues with low sound-speeds. The reflectivity (normal reflection coefficient) within the phantom is depicted in Fig. 2(b).

Reflectivity images reconstructed using the phase-shift and the globally optimized Fourier finite-difference methods are displayed in Figs. 2(c) and (d), respectively. A uniform sound-speed, obtained by averaging the slowness (reciprocal of the sound speed) of the numerical breast phantom in Fig. 2(a), is used in the phase-shift imaging. The original heterogeneous sound-speed distribution in Fig. 2(a) is utilized during reflectivity image reconstruction with the globally optimized Fourier finite-difference method, and the image is shown in Fig. 2(d). The image in Fig. 2(c) is blurred and contains a significant amount of artifacts. The image resolution and quality are greatly improved in Fig. 2(d) when ultrasound scattering is 


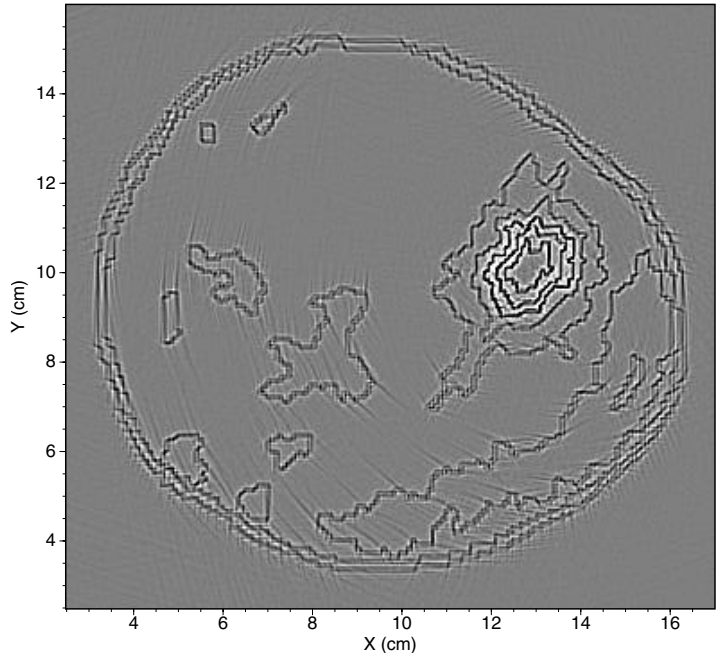

(a) Reflectivity image reconstructed with a uniform sound-speed

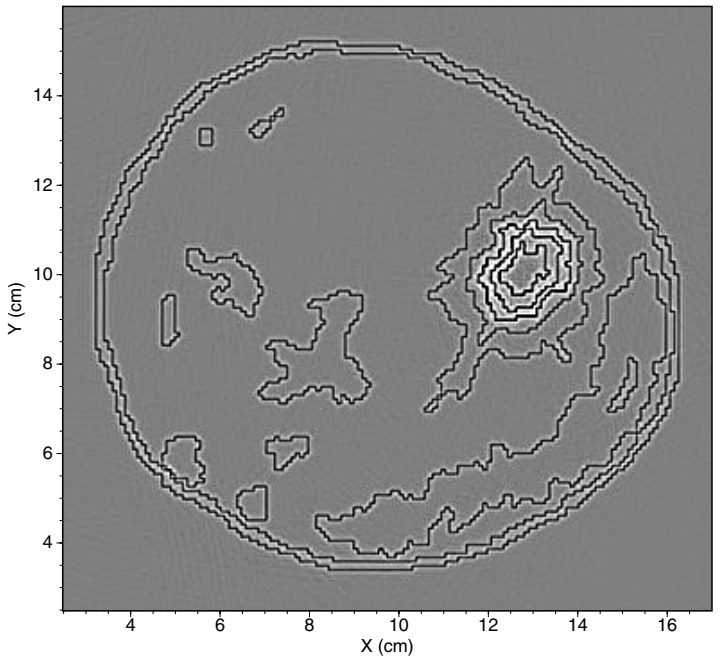

(c) Reflectivity image reconstructed with a heterogeneous sound-speed model Fig. 3(d)

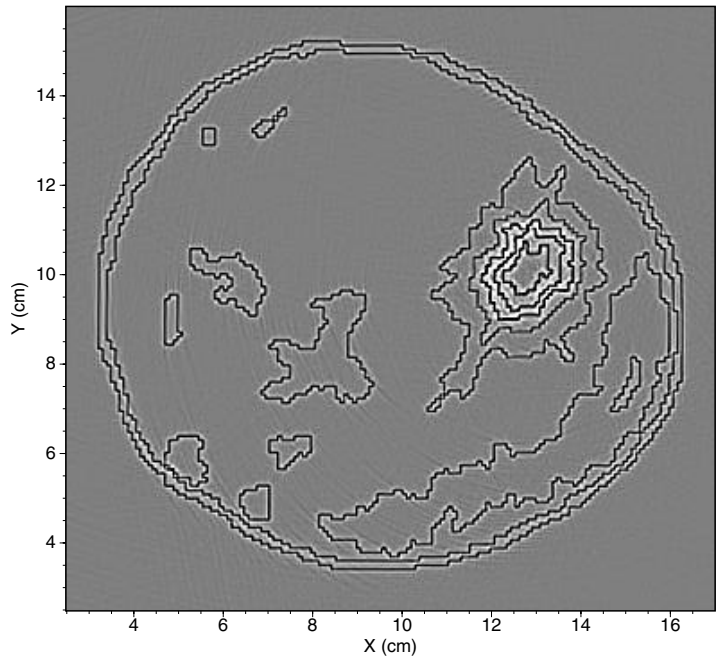

(b) Reflectivity image reconstructed with a heterogeneous sound-speed model in Fig. 3(c)

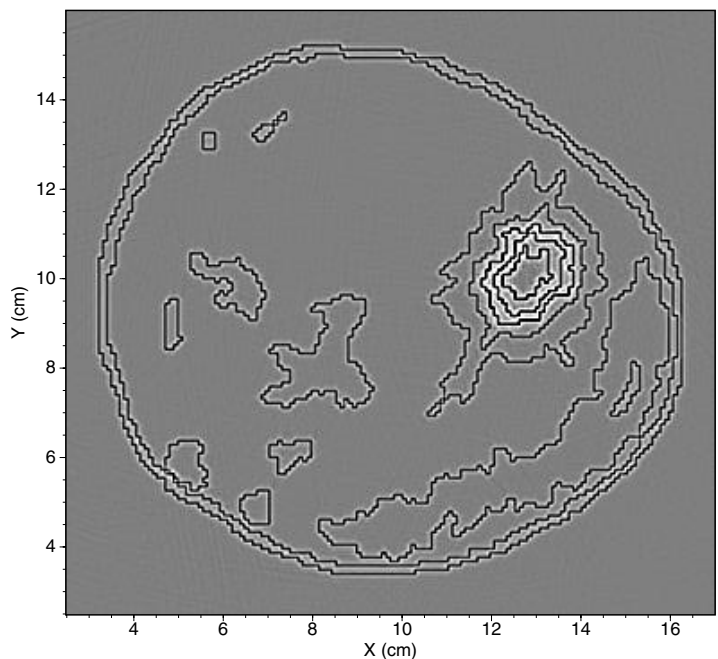

(d) Reflectivity image reconstructed with the correct sound-speed of the phantom in Fig. 3(a)

Figure 4: (a) Reflectivity image of the phantom in Fig. 3(a) reconstructed using the phase-shift method and a uniform sound-speed. Images in (b)-(d) are produced using the globally optimized Fourier finite-difference method and the heterogeneous sound-speed distributions in Figs. 3(c), (d), and (a), respectively.

accurately accounted for during imaging with the globally optimized Fourier finite-difference method. This demonstrates the importance of properly and accurately handling ultrasound scattering in ultrasound reflection imaging.

Next we use a numerical breast phantom shown in Fig. 3(a) to investigate the imaging capability of our globally optimized Fourier finite-difference method. The phantom contains heterogeneous breast tissues and a breast cancer with a higher sound-speed than its surrounding tissues. Figure 3(b) depicts the reflectivity within the phantom. Figures 3(c) and (d) are different results of sound-speed transmission tomography for the phantom in Fig. 3(a). They give low-resolution images of the phantom.

We conduct reflectivity image reconstructions using different sound-speed distributions of the numerical breast phantom in Fig. 3(a). Figure 4(a) is produced using a uniform sound-speed, and once again demonstrates that ultrasound scattering results in a blurred image with a lot of artifacts. The images in Fig. 4(b)-(d) are reconstructed using the globally optimized Fourier finite-difference method and the heterogeneous sound-speed models in Figs. 3(c), (d) and (a), respec- 


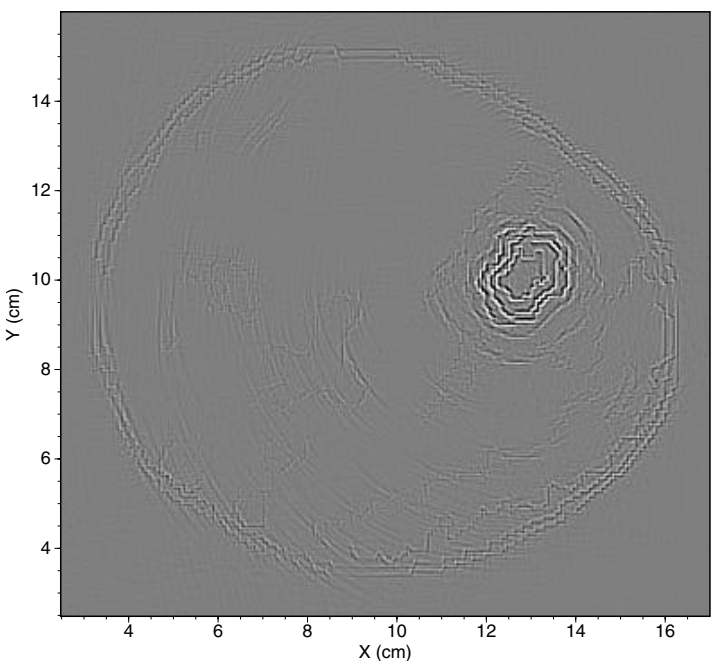

(a)

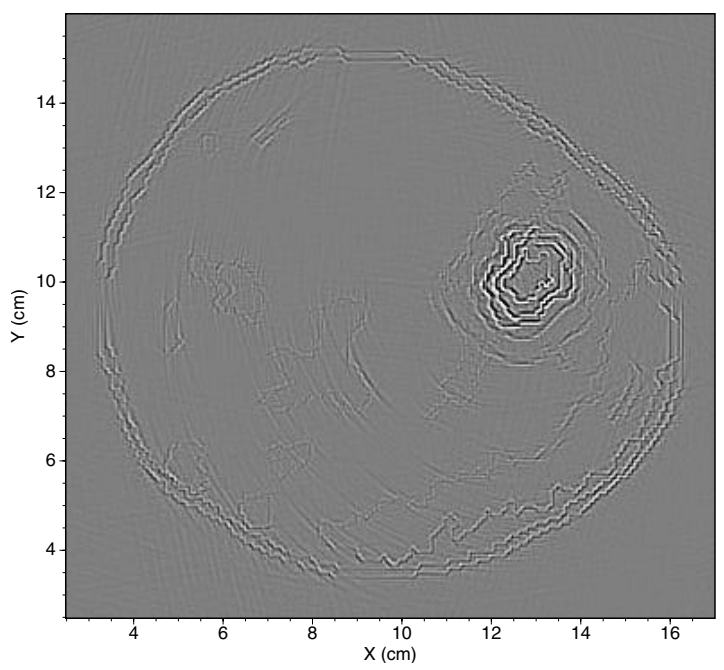

(c)

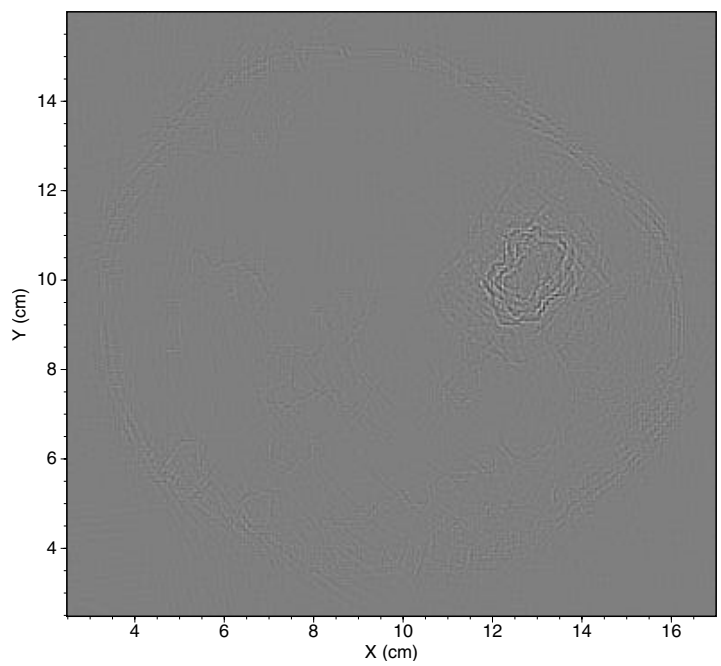

(b)

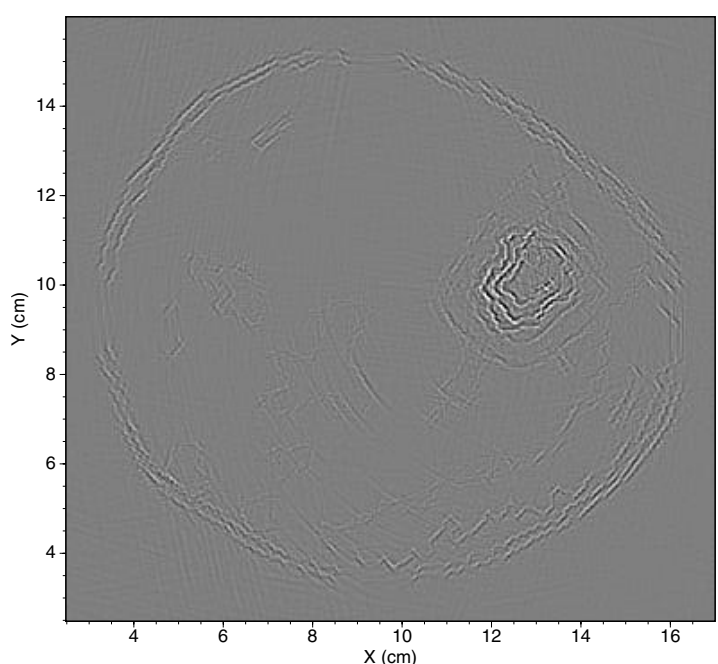

(d)

Figure 5: Comparison of the differences ("image artifacts") of reconstructed reflectivity images compared with the image shown in Fig. 4(d). Panels (a) and (b) are the differences between the images in Figs. 4(b) and (c) with that in Fig. 4(d), all obtained using the optimized propagator. Panels (c) and (d) are the corresponding differences of images obtained using the split-step Fourier method with the sound-speeds in Figs 3(c) and (d).

tively. These results demonstrate that it is essential to properly account for ultrasound scattering to produce high-resolution and high-quality ultrasound reflection images.

We investigate image artifacts caused by the propagator inaccuracy and the sound-speed discrepancy. As demonstrated in the numerical imaging examples in Figs. 2 and 4, it is necessary to properly and accurately accounting for ultrasound scattering during reflectivity image reconstruction to produce high-resolution and high-quality ultrasound images. This requires an accurate propagator for ultrasound wavefield inward continuation as well as an accurate sound-speed model of the breast. Reflectivity image artifacts (including image noise, incorrect image location and amplitudes) are caused not only by the propagator inaccuracy, but also by the discrepancies/errors in the sound-speed model used for image reconstruction.

Figure 4(d) is a reflectivity image reconstructed using the globally optimized Fourier finite-difference method and the correct sound-speed of the numerical breast phantom in Fig. 3(a). Therefore, it does not contain any image artifacts caused by the sound-speed error. We use this image as a standard in image comparison.

Figure 5 shows a comparison of differences between various reflectivity images and Fig. 4(d). The sound-speed model 
used in Figs. 5(a) and (c) is a less accurate one in Fig. 3(c), and that used in (b) and (d) is the more accurate one shown in Fig. 3(d). Comparing Fig. 5(b) with Fig. 5(a), and Fig. 5(d) with Fig. 5(c), it is obvious that reflectivity image artifacts decrease with increasing accuracy of the sound-speed tomography results. When the sound-speed error is large, comparison between Fig. 5(a) and Fig. 5(c) indicates that reflectivity images are similar to one another no matter which method is used for image reconstruction. That is, image artifacts caused by the sound-speed error are much stronger than those caused by the inaccuracy of imaging methods when the sound-speed error is large. When the sound-speed error is small, comparison between Fig. 5(b) and Fig. 5(d) demonstrates that image artifacts decrease with increasing the accuracy of the imaging method.

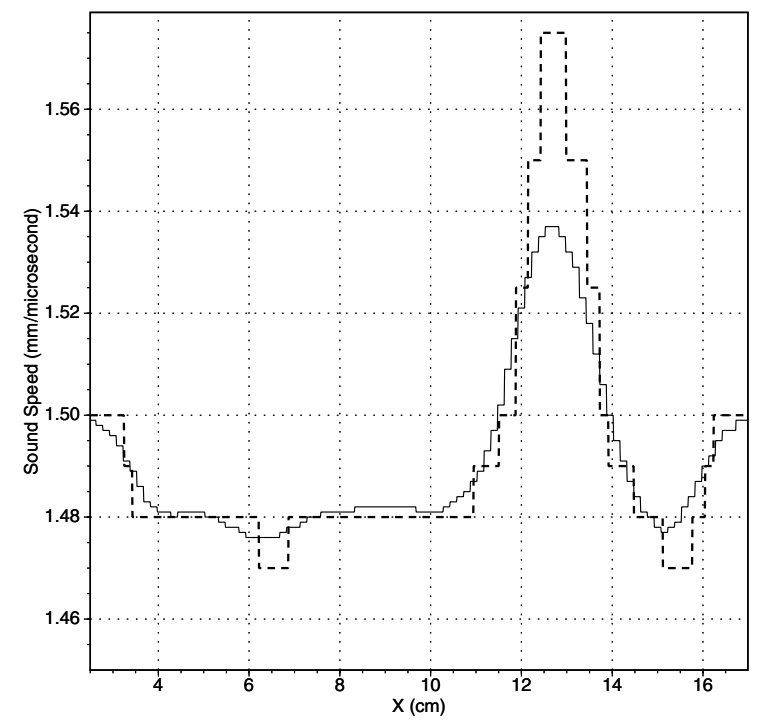

(a)

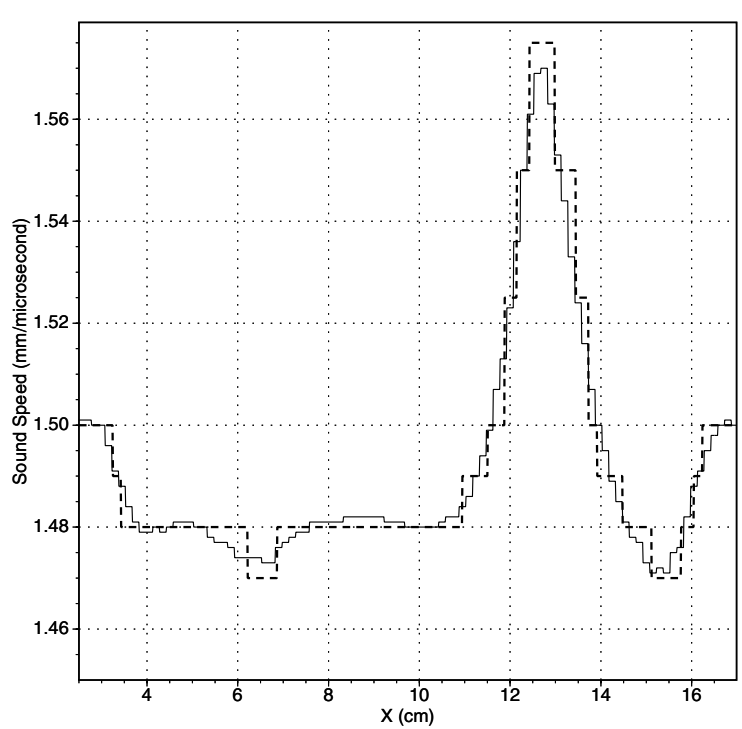

(b)

Figure 6: The cross-section profiles of sound speed at $y=9.8 \mathrm{~cm}$ for the phantom in Fig. 3. The dashed line in each panel is the correct sound-speed in Fig. 3(a). The solid lines in (a) and (b) are, respectively, the sound-speed cross-section profiles of tomography results in Figs. 3(c) and (d).

The sound-speed cross-section profiles displayed in Fig. 6(a) show large sound-speed errors in the tomography result shown in Fig. 3(c) with only two iterations. The sound-speed errors are particularly large around the cancer region. With ten iterations during sound-speed reconstruction, the sound-speed tomography result depicted in Fig. 6(b) is very close to the correct sound-speed of the phantom. Relative image differences in Fig. 7 give a quantitative comparison of image errors. It shows that the most significant image errors occur around the cancer region. The image errors in Figs. 7(a) and (c) are large due to large sound-speed errors as shown in Fig. 6(a). However, the difference between Fig. 7(a) and Fig. 7(c) is insignificant. When the sound-speed discrepancies are small (Fig. 6b), the errors/artifacts in the image obtained using the optimized method (Fig. 7b) are much smaller/weaker than that produced with the split-step Fourier method (Fig. 7d).

We reconstruct ultrasound reflectivity images of the numerical breast phantom in Fig. 3(a) using different sound-speed tomography results with 1-10 iterations during sound-speed tomography reconstructions. Then we calculate the total absolute image differences compared to that in Fig. 4(d), and the corresponding total absolute errors of sound speed in tomography results. Figure 8 depicts their relationships for the globally optimized Fourier finite-difference method and the split-step Fourier method. It clearly shows that the overall image error increases rapidly with the overall sound-speed error. The overall image error for the split-step Fourier method is larger than that for the optimized method for a given sound-speed error.

\section{CONCLUSIONS}

We have developed a globally optimized Fourier finite-difference method for ultrasound reflectivity imaging. The method is optimized for the entire range of sound-speed perturbations within the breast. It is much more accurate than the split-split 


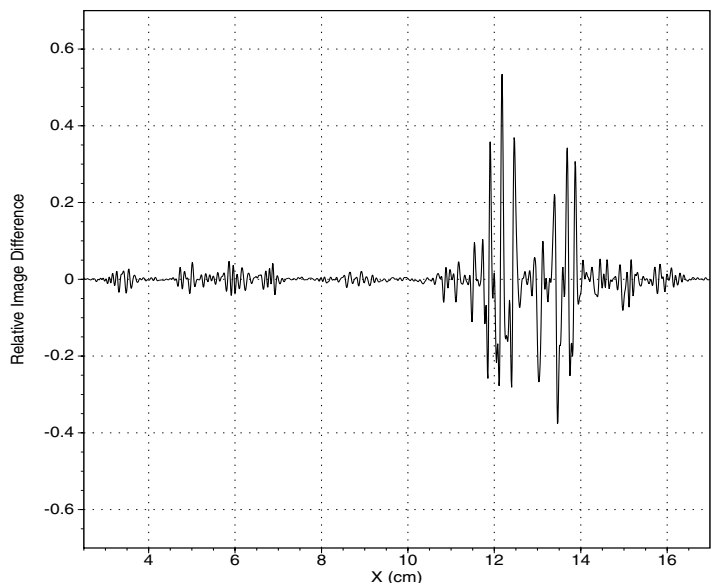

(a)

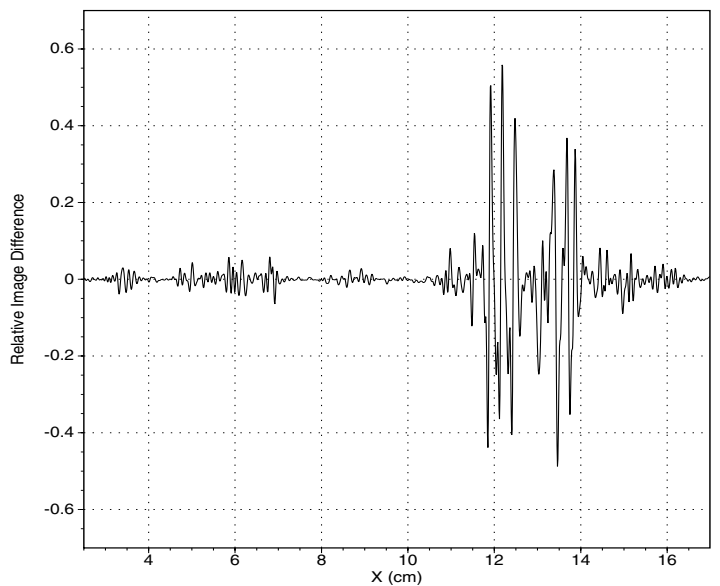

(c)

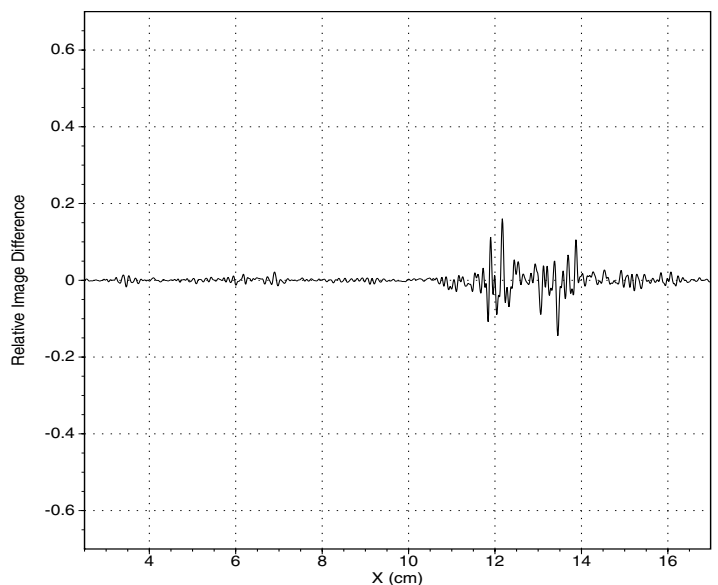

(b)

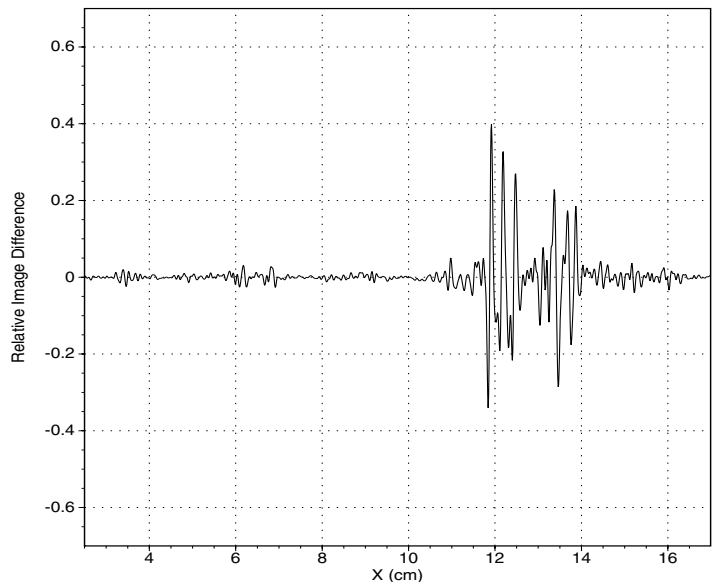

(d)

Figure 7: Panels (a)-(d) are, respectively, the relative image differences along the cross sections of the panels in Fig. 5 at $\mathrm{y}=9.8 \mathrm{~cm}$.

Fourier method for handling ultrasound scattering in the heterogeneous breast during reflectivity image reconstruction. We have also numerically demonstrated the importance of an accurate sound-speed model of the breast for reflectivity image reconstruction. The image error increases rapidly with increasing the sound-speed error. Both the accurate imaging method and the reliable sound-speed tomography result of the heterogeneous breast are critically important for high-resolution and high-quality ultrasound breast imaging. Our new optimized imaging method has great potential for clinical applications.

\section{ACKNOWLEDGMENT}

This work was supported through the U.S. DOE Laboratory-Directed Research and Development program at Los Alamos National Laboratory.

\section{REFERENCES}

[1] Kolb, T. M., Lichy, J., and Newhouse, J. H., "Occult cancer in women with dense breast: detection with screening us diagnostic yield and tumor characteristics," Radiology 207, 191-199 (1998).

[2] Sickles, E. A., "Breast imaging: From 1965 to the present," Radiology 215, 1-16 (2000).

[3] Houssami, N., Irwig, L., Simpson, J., McKessar, M., Blome, S., and Noakes, J., "Sydney breast imaging accuracy study: comparative sensitivity and specificity of mammography and sonography in young women with symptoms," American Journal of Roentgenology 180, 935-940 (2003). 


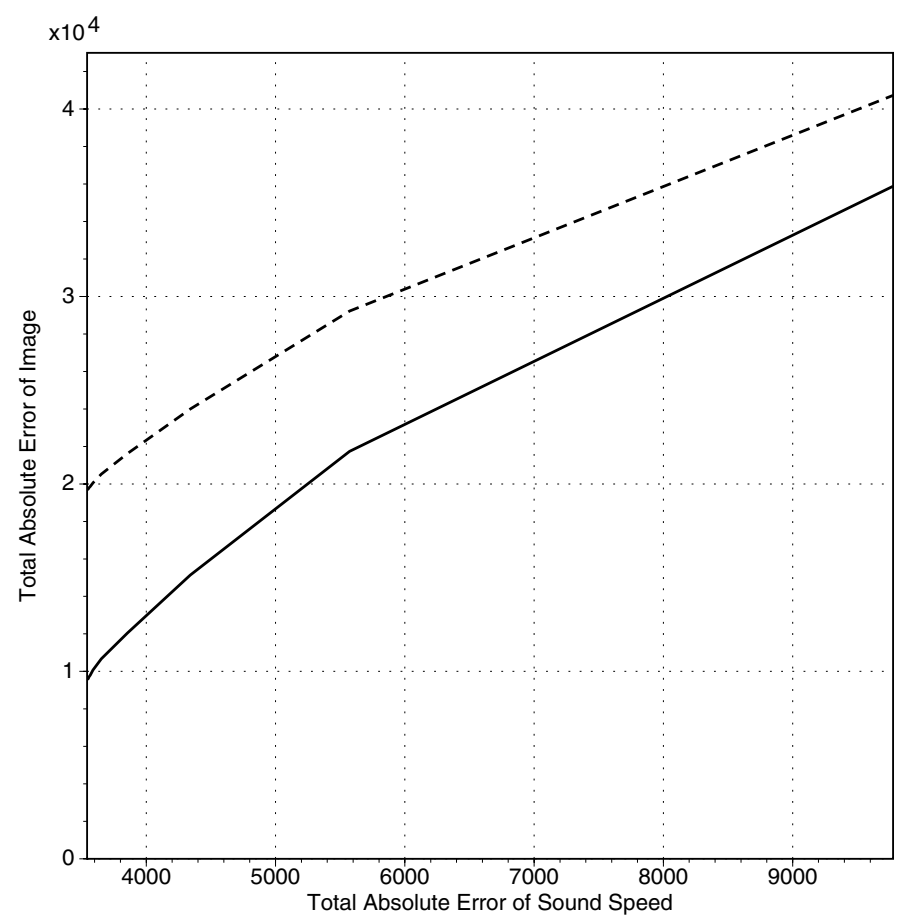

Figure 8: The total absolute error of the ultrasound reflection image versus the total absolute error of sound speed (in $\mathrm{mm} / \mu \mathrm{s}$ ) within the imaging region for the globally optimized Fourier finite-difference method (solid line) and the splitstep Fourier method (dashed line).

[4] Norton, S. J. and Linzer, M., "Ultrasonic reflectivity tomography: reconstruction with circular transducer arrays," Ultrasonic Imaging 2, 154-184 (1979).

[5] Schreiman, J. S., Gisvold, J. J., Greenleaf, J. F., and Bahn, R. C., "Ultrasound transmission computed tomography of the breast," Radiology 150, 523-530 (1984).

[6] Littrup, P. J., Duric, N., Azevedo, S., Chambers, D. H., Candy, J. V., Johnson, S., Auner, G., Rather, J., and Holsapple, E. T., "Computerized Ultradound Risk Evaluation (CURE) system: Development of combined transmission and reflection ultrasound with new reconstruction algorithms for breast imaging," Acoustical Imaging 26, 175-182 (2002).

[7] Stotzka, R., Gobel, G., and Schlote-Holubek, K., "Development of transducer arrays for ultrasoundcomputertomography," in [Proc. SPIE: Ultrasonic Imaging and Signal Processing], Walker, W. F. and Insana, M., eds., Proc. SPIE Medical Imaging 5035, 513-520, SPIE, Bellingham, Washington (2003).

[8] Duric, N., Littrup, P. J., Babkin, A., Chambers, D., Azevedo, S., Kalinin, A., Pevzner, R., Tokarev, M., Holsapple, E., Rama, O., and Duncan, R., "Development of ultrasound tomography for breast imaging: Technical assessment," Med. Phys. 32, 1375-1386 (2005).

[9] Waag, R. C. and Fedewa, R. J., "A ring transducer system for medical ultrasound research," IEEE Trans. Ultrason. Ferroelectr. Freq. Control 53, 1707-1718 (2006).

[10] Duric, N., Littrup, P., Poulo, L., Babkin, A., Pevzner, R., Holsapple, E., and Rama, O., "Detection of breast cancer with ultrasound tomography: First results with the computerized ultrasound risk evaluation (cure) prototype," Med. Phys. 34, 773-785 (2007).

[11] Andre, M. P., Janee, H. S., Martin, P. J., Otto, G. P., Spivey, B. A., and Palmer, D. A., "High-speed data acquisition in a diffraction tomography system employing large-scale toroidal arrays," International Journal of Imaging Systems and Technology 8, 137-147 (1997).

[12] Chambers, D. H. and Littrup, P., "Ultrasound imaging using diffraction tomography in a cylindrical geometry," in [Proc. SPIE: Ultrasonic Imaging and Signal Processing], Insana, M. and Walker, W. F., eds., Proc. SPIE Medical Imaging 4687, 412-420, SPIE, Bellingham, Washington (2002).

[13] Leach Jr., R. R., Azevedo, S. G., Berryman, J. G., Bertete-Aguirre, H. R., Chambers, S. H., Mast, J. E., Littrup, P., Duric, N., Johnson, S. A., and Wuebbeling, F., "Comparison of ultrasound tomography methods in circular geometry," 
in [Proc. SPIE: Ultrasonic Imaging and Signal Proceesing], Insana, M. and Walker, W. F., eds., Proc. SPIE Medical Imaging 4687, 362-377, SPIE, Bellingham, Washington (2002).

[14] Duric, N., Littrup, P., Holsapple, E. T., Babkin, A., Duncan, R., Kalinin, A., Pevzner, R., and Tokarev, M., "Ultrasound tomography of breast tissue," in [Proc. SPIE: Ultrasonic Imaging and Signal Processing], Walker, W. F. and Insana, M., eds., Proc. SPIE Medical Imaging 5035, SPIE, Bellingham, Washington (2003).

[15] Pratt, R. G., Huang, L., Duric, N., and Littrup, P., "Sound-speed and attenuation of the breast tissue using waveform tomography of transmission ultrasound data," in [Proc. SPIE: Physics of Medical Imaging], Hsieh, J. and Flynn, M. J., eds., Proc. SPIE Medical Imaging 6510, SPIE, Bellingham, Washington (2007).

[16] Quan, Y. and Huang, L., "Sound-speed tomography using first-arrival transmission ultrasound for a ring array," in [Proc. SPIE: Ultrasonic Imaging and Signal Processing], Emelianov, S. Y. and McAleavey, S. A., eds., Proc. SPIE Medical Imaging 6513, SPIE, Bellingham, Washington (2007).

[17] Huang, L. and Quan, Y., "Ultrasound pulse-echo imaging using the split-step fourier propagator," in [Proc. SPIE: Ultrasonic Imaging and Signal Processing], Emelianov, S. Y. and McAleavey, S. A., eds., Proc. SPIE Medical Imaging 6513, SPIE, Bellingham, Washington (2007).

[18] Huang, L., Duric, N., and Littrup, P., "Breast imaging with time-reversed ultrasound," in [Proc. SPIE: Ultrasonic Imaging and Signal Processing ], Emelianov, S. and Walker, W. F., eds., Proc. SPIE Medical Imaging 6147, 156-167, SPIE, Bellingham, Washington (2006).

[19] Huang, L., Duric, N., and Littrup, P., "Ultrasonic breast imaging using a wave-equation migration method," in [Proc. SPIE: Ultrasonic Imaging and Signal Processing ], Walker, W. F. and Insana, M., eds., Proc. SPIE Medical Imaging 5035, 432-439, SPIE, Bellingham, Washington (2003). 